\title{
Insured clients out-of-pocket payments for health care under the national health insurance scheme in Ghana
}

\author{
Patricia Akweongo ${ }^{1 *}$, Moses Aikins ${ }^{1}$, Kaspar Wyss ${ }^{2}$, Paola Salari $^{3}$ and Fabrizio Tediosi ${ }^{2}$
}

\begin{abstract}
Background: In 2003, Ghana implemented a National Health Insurance Scheme (NHIS) designed to promote universal health coverage and equitable access to health care. The scheme has largely been successful, yet it is confronted with many challenges threatening its sustainability. Out-of-pocket payments (OOP) by insured clients is one of such challenges of the scheme. This study sought to examine the types of services OOP charges are made for by insured clients and how much insured clients pay out-of-pocket.

Methods: This was a descriptive cross-sectional health facility survey. A total of 2066 respondents were interviewed using structured questionnaires at the point of health care exit in the Ashanti, Northern and Central regions of Ghana. Health facilities of different levels were selected from 3 districts in each of the three regions. Data were collected between April and June 2018. Using Epidata and STATA Version 13.1 data analyses were done using multiple logistic regression and simple descriptive statistics and the results presented as proportions and means.

Results: Of all the survey respondents $49.7 \%$ reported paying out-of-pocket for out-patient care while $46.9 \%$ of the insured clients paid out-of-pocket. Forty-two percent of the insured poorest quintile also paid out-of-pocket. Insured clients paid for consultation (75\%) and drugs (63.2\%) while 34.9\% purchased drugs outside the health facility they visited. The unavailability of drugs (67.9\%) and drugs not covered by the NHIS (20.8\%) at the health facility led to outof-pocket payments. On average, patients paid GHS33.00 (USD6.6) out-of-pocket. Compared to the Ashanti region, patients living in the Northern region were $74 \%$ less at odds to pay out-of-pocket for health care.

Conclusion and recommendation: Insured clients of Ghana's NHIS seeking health care in accredited health facilities make out-of-pocket payments for consultation and drugs that are covered by the scheme. The out-of-pocket payments are largely attributed to unavailability of drugs at the facilities while the consultation fees are charged to meet the administrative costs of services. These charges occur in disadvantaged regions and in all health facilities. The high reliance on out-of-pocket payments can impede Ghana's progress towards achieving Universal Health Coverage and the Sustainable Development Goal 3, seeking to end poverty and reduce inequalities. In order to build trust and confidence in the NHIS there is the need to eliminate out-of-pocket payments for consultation and medicines by insured clients.
\end{abstract}

Keywords: Ghana, Out-of-pocket, National health insurance scheme, Insured clients, Inequity, Access

\footnotetext{
*Correspondence: pakweongo@ug.edu.gh; akweongo@gmail.com

${ }^{1}$ School of Public Health, University of Ghana, P. O. Box LG13, Accra, Ghana

Full list of author information is available at the end of the article
} 


\section{Background}

Essential health care services remain unavailable to many people in need of care. Half of the world's population cannot still obtain essential health services and about 800 million people spend about $10 \%$ of their household budget on health care expenses, while over 100 million are further pushed into extreme poverty by paying for care out-ofpocket [1]. The proportion of households and patients paying-out-of-pocket for care is growing with about 808 million people estimated globally to have incurred catastrophic health spending in 2010 [1]. This continues to impose huge financial risks on households, especially vulnerable households, potentially pushing such households into a vicious cycle of poverty.

The declaration of Universal Health Coverage (UHC) as a policy for all countries comes timely as UHC seeks to ensure "all people can use the promotive, preventive, curative, rehabilitative and palliative health services they need, of sufficient quality to be effective, while ensuring that the use of these services does not expose the user to financial hardship" [2]. The demand for social protection schemes as a way of mitigating against the effect of out-of-pocket payments and poverty is growing as social protection schemes prove successful in fighting against poverty.

Social protection schemes either voluntary and/or mandatory have been adopted by middle- and lowincome countries with the aim of increasing equity in access to health services and providing financial risk protection, especially for the poor [3]. The introduction of financial protection schemes seeks to pool funds to protect individuals against catastrophic health expenditures. Social health insurance reduces household vulnerability to high out-pocket payments for health services in times of illness through reduction in direct medical costs and loss of income due to ill health [2] Sood et al. [4] indicated that poor households covered under these schemes tend to have reduced mortality, better access to care and reduce health care expenditures. Out-of-pocket payments by eligible households in this same study was also reduced by $64 \%$ for hospital admission fees [4].

Similarly, health care access increases for vulnerable groups covered under health insurance than for those not covered. Globally, evidence shows that social health insurance improves access to health care services $[3,5]$. There is also evidence that health insurance decreased out-pocket-payments between 16 and 18\% in Vietnam with more substantial decreases for low-income households [6-8]. However, out-of-pocket payments among the insured are still high in other countries and this affects access to health care [7].

Ghana introduced a national health insurance scheme (NHIS) in 2003 as a form of health financing mechanism. The NHIS aims at improving financial access to health care to all people living in Ghana irrespective of social status. Despite over a decade and half of the implementation of the NHIS, enrolment unto the scheme remains low. A published study reports coverage of the NHIS to be $35 \%$ [9]. A recent study found that being a member of NHIS significantly decreased the probability of unmet medical needs by $15 \%$ and that of incurring catastrophic out-of-pocket (OOP) health payments by $7 \%$ relative to no enrolment in the NHIS [10].

Over the years the NHIS has faced several challenges that threatens its sustainability. Amongst these challenges are; the huge benefit package, which covers over 95\% of the disease burden of Ghanaians has also been one of the highlights as it is observed to have significant implications for the financing of health care under the scheme. Moreover, there are several studies that have reported poor quality of health care under the scheme [11-13] a factor that is believed to contribute to the low level of enrolment among the populace. Worse yet, there is evidence that the insured are treated unfairly at health facilities, partly due to delays in reimbursement of payment to service providers. Therefore, providers give preference to patients that pay out-of-pocket [14] Also, it was reported that the currently uninsured perceived the quality of health care to be better than the currently insured in terms of waiting time, availability of medicines and so on [14].

The delays in payment of services provided to clients by providers is mainly due to inefficient financial flows. There are thus widespread unauthorized charges that insured clients are paying out-of-pocket which should not be the case. Macha et al. [15] reported Ghana to have the highest out-of-pocket payments of health care accounting for about $40 \%$ of total health care expenditure, followed by Tanzania at nearly $26 \%$ and South Africa at about $18 \%$. This, notwithstanding over $70 \%$ of both public and private health facilities are credentialed NHIS facilities in Ghana [16] .

Macha et al. [15], further reported that patients whether insured or not, paid out-of-pocket for medicines at pharmacies due to stock-outs in public health facilities or due to poor understanding of what they are entitled to as clients of the NHIS. The question however remains, what type of services are these unauthorized charges being made, who pays these charges and how much do they actually pay out-of-pocket?

\section{Methods}

\section{Study design}

This was a descriptive cross-sectional exit health facility survey. It was conducted among patients accessing outpatient care services at different levels of the health system in three regions of Ghana. The three regions selected were the Northern, Central and Ashanti based on the proportion of people who registered with the NHIS 
in 2017. The study chose these three regions purposively based on the proportion of NHIS membership in these regions. Ashanti region had the most registered number of clients, with moderate registration in Northern region and low registration in Central region. We also selected these regions because they each had a tertiary hospital that provided teaching, training and served as referral facilities for specialist care.

\section{Data collection methods}

Quantitative data collection approach was used to gather information about services patient paid for and how much they paid out-of-pocket. Patients were interviewed at the point of exit of the health care system using structured questionnaire. Data for the study was collected from April 2018 to June 2018.

\section{Sample size and sampling method}

An average of 688 out-patients were interviewed in each region. One district per region was first randomly selected and in each selected district, three health centres, the district hospital and two Community-based Health Planning and Services (CHPS) compounds were then randomly selected based on size of attendance at each of these health facilities. The number of patients interviewed at each level of the health system was proportional to the size of out-patient-department attendance of that facility for the previous year. For each day of the study, interviewees were conveniently sampled and interviewed as they exited the health facility from the dispensary. Since each interview lasted 30-35 min it meant that another interviewee could be only conveniently interviewed after every $30 \mathrm{~min}$. This was done daily until the sample size for each facility was achieved.

The number of OPD (Out-Patient Department) attendances at CHPS facilities was much lower than anticipated and thus more interviews were carried out at the health centre levels than in CHPS compounds. Interviews with patients at the teaching hospital in the Ashanti region could not be conducted as they required their own ethical review board review of the proposal and due to time constraints, the interviews were done at the district and regional hospitals in the Ashanti region.

\section{Data management and analysis}

Data was entered using Epidata and analyses were done using STATA Version 13.1. The study sought to establish the proportion of insured patients paying out-ofpocket for health care and to assess the determinants of paying out-of-pocket and the costs of care. The main outcome variable was the proportion of patients insured and paying out-of-pocket. Socio-economic status was measured using the wealth index where household assets and possessions of patients interviewed were used to construct the wealth index. Households were divided into five quintiles with first quintile representing the poorest household and the 5th quintile representing the least poor household. Data on household consumption expenditure was also gathered to calculate the proportion of household expenditure on health care. A multiple logistic regression was run to establish the association between being insured and paying out-of-pocket for health care. The type of services paid for are presented as proportions and percentages while the costs of health care and household consumption are presented as means and totals.

\section{Ethical considerations}

The protocol was reviewed and approved by the Ghana Health Service Ethics Review Committee (GHS-ERC: 012/03/18) before the commencement of data collection. Informed consent both written and oral was obtained from potential participants before the interviews were conducted. For minors, informed consent from parents or caregivers who accompanied them to the health facility was obtained. Informed consent was sought from all adult participants 18 years and above.

The interviewers explained to all study participants the purpose of the study, study procedure, right to withdraw and measures put in place to ensure confidentiality. Participants were told data will be reported in an aggregated format and anonymity will be ensured in storage and publication of the results of the study.

\section{Results}

\section{Socio-demographic and economic characteristics of respondents}

Of the 2066 respondents who participated in the survey $47 \%$ were in the age bracket $18-49$ years (Table 1 ). There were more females $76.7 \%$ (1591) who accessed health care than males. Most respondents were Akan $61.4 \%$ (1273). This was not surprising, as the Ashanti and Central regions are Akan speaking communities. A little over 50\% (1099) were married and 9.4\% (195) of the respondents were living together. The average household was size 4.7 (Table 1).

Less than a third (30.2\%) of the participants completed Junior High School with 29.3\%(605) without any formal education (Table 2). Those with secondary or higher education were $29.7 \%$ (614). Thus, it is not surprising that $26.2 \%$ (541) reported being unemployed and with $20.5 \%$ (432) in the sales and services industry. Those working in the agriculture sector were 13.7\% (282). Those who had retired from active service or aged were $11.7 \%$ (241).

Of the 2066 respondents, $52.7 \%$ (1094) received remittances from relatives or friends (Table 2). Cash remittances constituted $67.6 \%$ (738). Most remittances were 
Table 1 Socio-demographic and economic background of respondents

\begin{tabular}{|c|c|c|}
\hline & Number & Percentage (\%) \\
\hline \multicolumn{3}{|l|}{ Demographic characteristic } \\
\hline \multicolumn{3}{|l|}{ Age group (Years) } \\
\hline$<18$ & 651 & 31.4 \\
\hline $18-49$ & 967 & 47.1 \\
\hline $50-69$ & 301 & 14.5 \\
\hline $70+$ & 147 & 7.1 \\
\hline Total & 2066 & 100.0 \\
\hline \multicolumn{3}{|l|}{ Sex: } \\
\hline Female & 1583 & 76.6 \\
\hline Male & 482 & 23.4 \\
\hline Total & 2066 & $100^{\prime} 0$ \\
\hline \multicolumn{3}{|l|}{ Ethnicity: } \\
\hline Akan & 1273 & 61.4 \\
\hline Mole-Dangme & 680 & 32.9 \\
\hline Other & 82 & 4.0 \\
\hline Foreign & 31 & 1.5 \\
\hline Total & 2066 & 100.0 \\
\hline \multicolumn{3}{|l|}{ Marital Status: } \\
\hline Married & 1099 & 53.2 \\
\hline Single-never married & 504 & 24.4 \\
\hline Divorce-separated & 81 & 3.9 \\
\hline Widowed & 187 & 9.1 \\
\hline Living together & 195 & 9.4 \\
\hline Total & 2066 & 100.0 \\
\hline \multicolumn{3}{|l|}{ Socio-economic characteristic } \\
\hline Level of education: & 605 & 29.3 \\
\hline Never been to school & 605 & 29.3 \\
\hline Primary & 233 & 10.8 \\
\hline Junior High School/Middle & 624 & 30.2 \\
\hline Senior High School/O \& A Level & 398 & 19.3 \\
\hline Tertiary & 216 & 10.4 \\
\hline Total & 2066 & 100.0 \\
\hline Main occupation: & 153 & 7.4 \\
\hline Agriculture & 282 & 13.7 \\
\hline Professional/secretarial & 153 & 7.4 \\
\hline Sales/services & 432 & 20.5 \\
\hline Skilled manual craftsmanship & 198 & 9.6 \\
\hline Unskilled manual labour & 125 & 6.1 \\
\hline Unemployed & 541 & 26.2 \\
\hline Others (i.e., aged/retired/students) & 339 & 16.5 \\
\hline Total & 2061 & 100.0 \\
\hline \multicolumn{3}{|l|}{ Remittance from relatives: } \\
\hline Received remittance & 1094 & 52.7 \\
\hline
\end{tabular}

Table 1 Socio-demographic and economic background of respondents (Continued)

\begin{tabular}{lll}
\hline & Number & Percentage (\%) \\
\hline Remittance type: & 738 & 67.6 \\
Cash Remittance & 353 & 32.4 \\
Gifts/in-kind & 1091 & 100.0 \\
Total & & \\
Remittance frequency & 323 & 30.7 \\
Weekly/daily & 249 & 23.0 \\
Monthly & 501 & 46.3 \\
Occasionally & 1073 & 100.0 \\
Total & & \\
Monthly income (GHS $\left.{ }^{a}\right)$ & 375 & 31.0 \\
$<150$ & 290 & 24.0 \\
151-300 & 127 & 10.5 \\
301-450 & 146 & 12.1 \\
$451-600$ & 270 & 22.4 \\
$600+$ & 1208 & 100.0 \\
Total & &
\end{tabular}

received occasionally. The mean monthly income was GHS618.46 (USD123.69) with a median income of GHS300.00 (USD60) for those who reported actively working. Less than a third (31\%) of the working population earned between GHS8.00 and GHS150.00 (USD30). Another 24\% earned between GHS151-300 (USD30.260 ), showing that $55 \%$ of the respondents earned below the minimum monthly wage of GHS 320.00 (USD64.00) for Ghana [17]. The mean monthly household recurrent expenditure of GHS678.00 (USD135.60) was similar to mean monthly income reported by the respondents. Non-recurrent expenditure was slightly higher than the mean monthly recurrent expenditure.

\section{Socio-demographic and economic characteristics by region}

In all the three regions, most of the respondents were in the 18-49 years age category (Table 2). Those under 5 years of age and less than 17 years were on average $30 \%$ (208) in the Ashanti region, 26.6\% (181) in Central region and over $37 \%$ (255) in the Northern region. This group is exempted from paying NHIS premium thus benefiting from free health care services. Though those 6-17 of school going age are exempted from premium payment, $56.2 \%$ of them paid for care OOP. Over $70 \%$ of the respondents in each region were females. Women in their reproductive age and who are pregnant are also exempted from paying national health insurance premium. There were variations in the levels of education 
Table 2 Socio-demographic and economic background of respondents by region

\begin{tabular}{|c|c|c|c|}
\hline \multirow[t]{2}{*}{ Items } & \multicolumn{3}{|c|}{ Study regions } \\
\hline & Ashanti (\%) & Central (\%) & Northern (\%) \\
\hline \multicolumn{4}{|l|}{ Age: } \\
\hline$<18$ & 30.0 & 26.6 & 37.9 \\
\hline $18-49$ & 41.9 & 47.1 & 51.2 \\
\hline $50-59$ & 10.8 & 9.2 & 3.3 \\
\hline $60-69$ & 8.8 & 7.8 & 3.6 \\
\hline $70+$ & 8.5 & 9.3 & 4.0 \\
\hline Total (n) & 692 & 688 & 688 \\
\hline \multicolumn{4}{|l|}{ Sex: } \\
\hline Female & 73.1 & 77.1 & 79.9 \\
\hline Male & 26.8 & 22.9 & 20.1 \\
\hline Total (n) & 692 & 688 & 683 \\
\hline \multicolumn{4}{|l|}{ Level of education: } \\
\hline Never been to school & 23.6 & 15.0 & 49.4 \\
\hline Primary & 10.3 & 13.4 & 8.7 \\
\hline Junior High School/Middle & 34.3 & 43.3 & 13.5 \\
\hline Senior High School/O \& A Level & 22.1 & 16.9 & 19.1 \\
\hline Tertiary & 9.7 & 12.3 & 9.3 \\
\hline Total (n) & 691 & 687 & 687 \\
\hline \multicolumn{4}{|l|}{ Occupation: } \\
\hline Agriculture & 4.4 & 17.3 & 21.0 \\
\hline Professional/secretarial & 10.1 & 8.1 & 5.4 \\
\hline Sales/Services & 23.1 & 19.1 & 22.5 \\
\hline Skilled manual craftsmanship & 10.7 & 12.2 & 7.4 \\
\hline Unskilled manual labour & 6.6 & 10.6 & 2.1 \\
\hline Unemployed & 29.5 & 22.0 & 31.1 \\
\hline Other (i.e., retired) & 15.7 & 10.7 & 10.6 \\
\hline Total $(n)$ & 637 & 654 & 672 \\
\hline \multicolumn{4}{|l|}{ Wealth quintiles } \\
\hline Poorest & 2.2 & 16.7 & 45.5 \\
\hline Very poor & 9.8 & 28.7 & 22.1 \\
\hline Poor & 23.2 & 18.7 & 17.7 \\
\hline Less poor & 31.2 & 17.8 & 8.9 \\
\hline Least poor & 33.6 & 18.1 & 5.7 \\
\hline Total $(n)$ & 682 & 663 & 560 \\
\hline \multicolumn{4}{|l|}{ Monthly income $\left(G H S^{a}\right)$ : } \\
\hline$<150$ & 27.3 & 32.7 & 32.7 \\
\hline $151-300$ & 29.4 & 21.6 & 21.7 \\
\hline $301-450$ & 10.1 & 12.0 & 9.2 \\
\hline $451-600$ & 14.2 & 13.1 & 8.9 \\
\hline $600+$ & 19.1 & 20.3 & 27.6 \\
\hline Total $(n)$ & 367 & 449 & 392 \\
\hline
\end{tabular}

a - Ghanaian local currency, Ghana cedis 
by region. Close to $50 \%$ (339) of respondents in the Northern region had never been to school compared to 23.6\% (163) in the Ashanti region and 15\% (103) in the Central region (Table 2).

The socio-economic status of respondents in each region showed $45.5 \%$ (255) of those in the Northern region to be in the poorest quintile. The Ashanti region reported more patients in the least poor quintile (33.6\%) than in Central (18.1\%) and Northern regions (5.7\%). These differences were statistically significant. However, monthly earnings did not vary much by region. Most participants earned between GHS8-150 (USD1.6030.00..) and GHS151-300 (USD30.20-60.00.) per month (Table 2).

\section{Utilization of health care services by respondents}

Most patients who participated in this survey visited in the last 6 months the government hospital 57.5\% (1187) and government health centres $38.2 \%$ (790) for health care services (Table 3). The choice of a health facility was based on three main factors; proximity $40.9 \%$ (845) of the facility to the patient, NHIS credentialed facility $29.6 \%$ (611) and availability of doctors $21.5 \%$ (444). Patients reported using hospitals and health centres more for all levels of severity of illness $41.4 \%$ (855) with only about $18.6 \%$ (384) using these facilities only during severe illness. For severity of illness patients were asked to indicate the stage of the illness (Mild, moderate, severe and very severe) at which they make decision to visit a particular facility to seek care. Patients who presented at the out-patient-department with fever and malaria like signs and symptoms were $47.5 \%$ (981) and nearly a quarter of patients $23.4 \%$ (485) were there for a review of their condition recommended by the health provider from a previous visit. (Table 3).

\section{Type of health services provided to patients at the out-} patient-department

Over 76.6\% (1583) of patients reported having consultation with the health care provider while $14.8 \%$ (306) came for a resupply of their medication (Table 4). After consultation, 87.3.\% (1382) were prescribed at least one drug. Twenty-nine percent (459) were prescribed two drugs and $25.8 \%$ (408) were prescribed three drugs. There was an observed pattern in the number of drugs prescribed and number of these drugs that were supplied by the pharmacy or dispensary of the health facility the patient visited. On average $75.6 \%$ (686) of all drugs prescribed were supplied by the pharmacy or dispensary of the facility the patient visited (Table 4).

However, of those who were prescribed with two drugs, $77.1 \%$ (354) of these drugs were supplied by the health facility they obtained care from. For those who were prescribed three drugs, $79.5 \%$ (365) received all the
Table 3 Health Care Services Accessed by Patients

\begin{tabular}{lll}
\hline Utilization of health care service & Number & Percentage (\%) \\
\hline Usual Care source & 790 & 38.2 \\
Health Centre & 1187 & 57.5 \\
Government-Hospital & 19 & 0.9 \\
Pharmacy-drug & 70 & 3.4 \\
Private-facility & 2066 & 100 \\
Total & & \\
Reason for choice & 845 & 40.9 \\
Proximity & 62 & 3.0 \\
Lab & 104 & 5.0 \\
Drugs available & 611 & 29.6 \\
NHIS Credential & 444 & 21.5 \\
Medical Doctors available & 2066 & 100 \\
Total & 2066 \\
Level of Severity of illness for Choice of Health Facility \\
Severity of illness & 384 & 18.6 \\
Moderate/mild & 827 & 40.0 \\
All levels of severity & 855 & 41.4 \\
Total & 2066 & 100 \\
Signs and symptoms presenting & & \\
Fever-malaria like & 981 & 47.5 \\
Difficult-in-breathing & 203 & 9.8 \\
ENT-toothache & 115 & 5.6 \\
Injury & 104 & 5.1 \\
Review & 485 & 23.4 \\
Eye infection & & 100 \\
Hypertension-diabetes & & \\
Skin Infections & & \\
Total & & \\
\hline
\end{tabular}

three drugs prescribed from the health facility. Of those prescribed with four drugs only $70.2 \%$ (188) were supplied with all four drugs (Table 4) in the health facility they obtained care from. Thus, close to a quarter of all the drugs prescribed were not supplied by the health facility the patients visited.

\section{Insurance status of patients}

Many respondents in this survey held a valid NHIS card at the time of the interview. The proportion of all patients with a valid NHIS card was 90.3\% (1722/1907) and a similar pattern was observed at the regional levels (Fig. 1). Northern region reported the highest NHIS coverage of $94 \%$ and the least $(87.9 \%)$ coverage was observed in the Ashanti region (Fig. 1). The proportion of males with valid NHIS card was $87.4 \%$ (368) while the proportion of females with valid NHIS card was $91.1 \%$ 
Table 4 Type of Services Accessed by Patients

\begin{tabular}{lll}
\hline Service Provision & Number & Percentage (\%) \\
\hline Services received at Facility & 68 & 3.3 \\
Maternal-child & 1583 & 76.6 \\
Consultation & 44 & 2.1 \\
Laboratory & 306 & 14.8 \\
Drugs & 32 & 1.6 \\
ENT-Eye-tooth & 33 & 1.6 \\
Hospital Admission & 2066 & 100 \\
Total & 201 & 12.7 \\
Number of Drugs Prescribed Among those who consulted \\
No drug prescribed & 247 & 15.6 \\
One drug prescribed & 459 & 29.0 \\
Two drugs prescribed & 408 & 25.8 \\
Three drugs prescribed & 268 & 16.9 \\
Four or More drugs prescribed & 1583 & 100 \\
Total & & \\
Number of drugs prescribed and number received by clients with \\
$\mathbf{2}$ or more drugs & 354 & 77.1 \\
Received All 2 drugs prescribed & 354 & 79.5 \\
Received All 3 drugs prescribed & 365 & 70.2 \\
Received All 4 drugs prescribed & 188 & 75.6 \\
Average of all drugs received & 686 & \\
\hline
\end{tabular}

(1349) and these differences were statistically significant $(P<0.000)$.

\section{Out-of-pocket payment for health services}

Close to $49.7 \%$ (1027) of all patients reported paying for health care at the health facility they visited. Sixty-four percent (657) paid consultation fee and 34.2\% (326) paid for drugs (Table 5). Whereas some patients incurred cost that otherwise should have been paid for by the NHIS, other costs were co-payments.

Of those who paid for these services, $42.5 \%$ (441) did not get a receipt for the services paid for. Patients reported ever paying for services at the same health facilities. Close to $48 \%$ (456) reported ever paying for these services in previous visits to the health facilities. For the most recent payments, $77.4 \%$ (322) paid weeks ago before this survey (Table 5).

Out-of-pocket payment for health care services occurred at all level of health facilities. On average close to $41 \%$ (441) of patients who visited the CHPS and $43 \%(881)$ health centres paid for health care while an average of $53 \%$ of those who visited district, regional and tertiary (teaching training hospitals) health facilities also paid out-of-pocket for health care.
Out-of-pocket payments at district and regional hospitals were slightly higher than those of tertiary hospitals but the differences were not statistically significant $(P>0.05)$.

Of all drugs prescribed, 34.9\% (717) were purchased outside the health facility visited (Table 5). The main reasons why the drugs were not supplied by the health facilities patients visited were: unavailability of the drug $67.9 \%$ (487) and the drugs prescribed not being covered by the NHIS $20.8 \%$ (142).

Most patients (66.5\%) paid between GHS1.00 (USD0.20) and GHS20.00 (USD4.00) for consultation and drugs (Table 5).

\section{Out-of-pocket payment for health services by insured clients}

Ninety percent of patients (1722) who accessed health care at all health facilities in the regions had a valid NHIS card and $8 \%$ (153) had a valid NHIS card but could not show it at the time of the survey (Table 6).

Insured clients who paid for health care were $46.9 \%$ (803) compared to $49.7 \%$ (89) of those who reported not having enrolled with the NHIS. This difference was not statistically significant .

Of those who held a valid NHIS card that was seen, $75.3 \%$ (1295) paid for consultation and 63.2\% (1079) paid out of pocket for drugs, and $26 \%$ paid for both consultation and drugs (Table 6). Of those with valid NHIS cards who could not present them, 68.5\% (102) paid for consultation and 75\% (111) paid for drugs (Table 6).

The proportion of NHIS clients in this study earning income was $56 \%$ (998). The median monthly income of NHIS clients was GHS300.00 (USD60.00) while the mean income was GHS574.72 (USD114.94). However, over $55.6 \%$ of NHIS members earned in the range of GHS8.00 to GHS300.00 (Table 6). The average monthly health care costs paid per client was GHS33.1 representing $11 \%$ of the median income of GHS300.00 (USD60.00) of patients.

NHIS clients paying-out-of-pocket for health care varied by region with $62.4 \%$ (431) of those from the Ashanti region paying out of pocket with much lower payments observed in the Central 48.2\% (335) and Northern regions $35.2 \%$ (238) and these differences were statistically significant $(P<0.001)$ as shown in Fig. 2.

There were regional differences in type of services paid for. Most patients in the Ashanti and Central regions paid more for consultation while patients in the Northern region paid more for drugs (Fig. 3). The differences in type of services paid out-of-pocket by region were statistically significant $(P<0.001)$.

The distribution of out-of-pocket payments by socioeconomic index showed that the proportion of the poorest that are paying out-of-pocket is similar to that of the least poor. Using both the wealth index generated using household assets and possessions and the monthly 


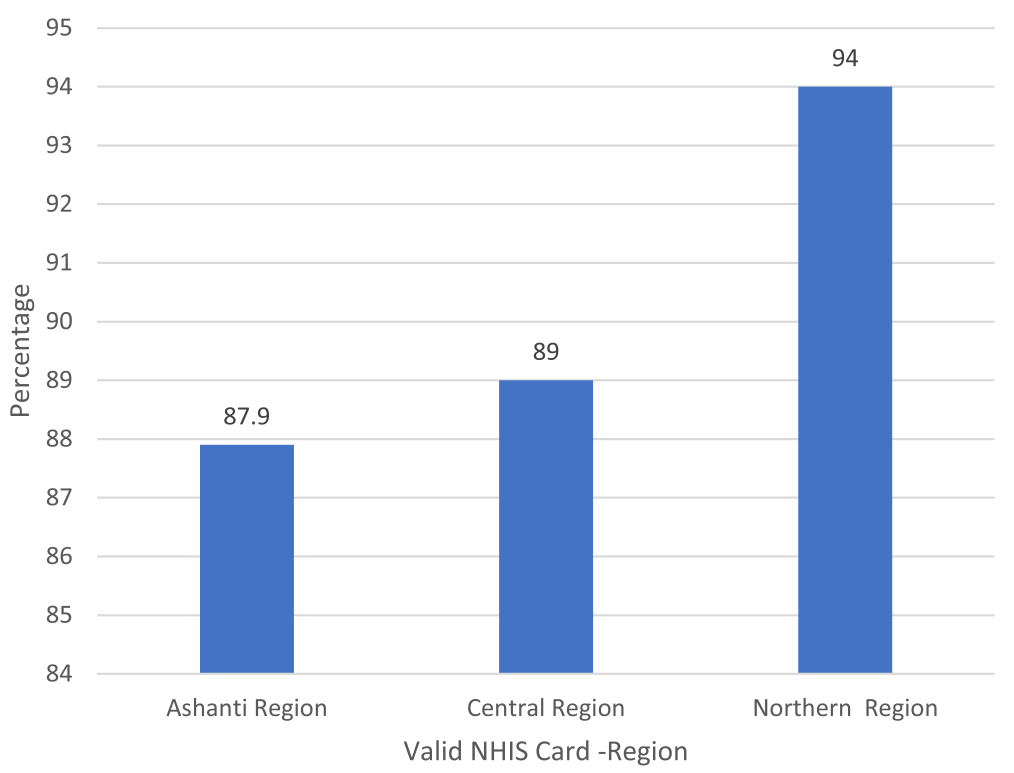

Fig. 1 Proportion of Patients with Valid NHIS Card by Region

household expenditure, both indices showed that $42 \%$ of the poorest quintile paid out-of-pocket and a similar proportion $43 \%$ of the least poor quintile paid out-ofpocket (Fig. 4) for health care. Thus, though the average paid out-of-pocket was GHS33.00 with 55\% paying in the range of GHS1.00-20.00 (USD0.20-USD4.00) and GHS21.00-40.00, (USD4.2-8.00) many of the poor earned between GHS8.00-150.00 (USD1.6-30.00). Thus, the out-of-pocket payments are borne more by the poorest quintile who earned in this range.

\section{Multiple logistic regression of the determinants of out-of- pocket payments for health care}

Sex, age, the level of education were not statistically significant determinants of out-of-pocket payments at the 5\% level of significance (Table 7). Region was a statistically significant determinant of out-of-pocket payments. Thus, compared to the Ashanti region, patients living in the Central region had $64 \%$ reduced odds (CI: $0.25-0.50$ ) of paying out-of-pocket for health care. Similarly, those living in the Northern region had about $74 \%$ reduced odds of paying out-of-pocket compared to patients in the Ashanti region. The odds of paying out-of-pocket increased significantly (33.97; 12.96-89.09) for those receiving laboratory investigation and consultation (12.09; 5.28-27.67). Also, the odds of paying out-of-pocket for health care services were 1.55 times higher for poor households than the poorest households whereas the odds of paying out-of-pocket for health care services were 2.08 times higher for the least poor households than the poorest households (Table 7).

\section{Discussion}

The objectives of this study was to determine the services NHIS clients pay for, the actual amounts clients paid out-of-pocket, those paying out-of-pocket and the determinants of out-of-pocket payments. Out-of-pocket payments are occurring at all the levels of health facilities. In lower level health facilities, patients made outof-pocket payment as much as $40 \%$ and in hospitals as much as $53 \%$. Close to $47 \%$ of NHIS clients with valid card reported out-of-pocket payments for Out-PatientDepartment (OPD) services in this study. Over twothirds of these patients presented with fever and malaria-like signs and symptoms. This is consistent with the report of Ghana Health Service where malaria was the leading cause of admission in hospitals nationwide in 2018 [18] .

Clients pay for consultation (75\%) and drugs (68.5\%), the basic services that NHIS covers. The average amount they paid out-of-pocket is GHS33.00. Nonetheless, 55\% pay between GHS1.00 and GHS40.00. This level of outof-pocket payment is confirmed in previous studies where NHIS clients paid between an average of GHS1317.50 out-of-pocket when seeking treatment $[13,19]$ Similarly, a reported $7-18 \%$ of insured households incurred catastrophic health expenditure in the Eastern and Central regions of Ghana [20] Moreover, in this study as many as $48.6 \%$ of clients report ever paying for health care in previous visits, further implying that paying out-of-pocket does occur. Patients pay $11 \%$ of their median monthly income of GHS300.00 as out-of-pocket payments. As much as $42 \%$ of the poorest quintile and 
Table 5 Out-of-pocket payments for health care by All patients

\begin{tabular}{|c|c|c|}
\hline Payment for Health Care & Number & $\begin{array}{l}\text { Percentage } \\
\text { (\%) }\end{array}$ \\
\hline Paid for services & 1027 & 49.7 \\
\hline \multicolumn{3}{|l|}{ Services paid for } \\
\hline Laboratory investigations & 44 & 4.4 \\
\hline Drugs & 326 & 32.4 \\
\hline Consultation & 657 & 64.0 \\
\hline Total & 1027 & 100 \\
\hline \multicolumn{3}{|l|}{ Cost of care paid for (GHS) } \\
\hline $1-20$ & 683 & 66.5 \\
\hline $21-40$ & 177 & 17.2 \\
\hline $41-60$ & 77 & 7.5 \\
\hline $61-80$ & 16 & 1.6 \\
\hline $81-100$ & 74 & 7.2 \\
\hline Total & 1027 & 100 \\
\hline \multicolumn{3}{|l|}{ Receipts received for services paid for } \\
\hline No receipts given & 441 & 42.5 \\
\hline Receipts for all services & 379 & 36.6 \\
\hline Receipts for some services & 217 & 21.0 \\
\hline Total & 1027 & \\
\hline Ever paid for services & 456 & 47.7 \\
\hline \multicolumn{3}{|l|}{ Most recent payment } \\
\hline Days ago & 66 & 15.9 \\
\hline Weeks ago & 322 & 77.4 \\
\hline Months ago & 28 & 6.7 \\
\hline Total & 456 & 100 \\
\hline $\begin{array}{l}\text { Proportion of drugs prescribed patient } \\
\text { purchased outside health facility patient } \\
\text { visited }\end{array}$ & 717 & 34.9 \\
\hline \multicolumn{3}{|l|}{ Reason for purchasing drug } \\
\hline Drug unavailable at facility & 487 & 67.9 \\
\hline Not covered by NHIS & 149 & 20.8 \\
\hline Expensive at facility & 7 & 1.0 \\
\hline Don't know & 74 & 10.3 \\
\hline Total & 717 & 100 \\
\hline \multicolumn{3}{|l|}{ Facility Type and out-of-pocket Payments } \\
\hline CHPS & 91 & 40.7 \\
\hline Health Centre & 881 & 42.7 \\
\hline District Hospital & 864 & 54.6 \\
\hline Tertiary & 226 & 51.8 \\
\hline
\end{tabular}

$43 \%$ of the least poor both pay out-of-pocket for health care, yet the poorest earn less than the current monthly minimum wage in Ghana (GHS320.00).

There were regional differences in out-of-pocket payments with those in the Ashanti region reporting more out-of-pocket payments than those in the Northern region. The variation reported between the two regions could be attributed to the general poverty level variations between these two regions [21] Generally, the Ashanti region is considered to be more economically vibrant than the Northern region, a situation that puts the people in Ashanti region in a better economic position than their counterparts in the Northern region. Therefore, service providers will be more inclined to expect out-of-pocket payments from the clients in Ashanti compared with clients in the Northern region.

Paying for services is attributed to unavailability of prescribed drugs or prescribed drugs not covered by the NHIS. The hospitals usually procure medical supplies and drugs on credit and pay suppliers later. This is a common practice based on the original design of the NHIS which reimburses service providers some weeks after service provision, when claims have been submitted to the National health insurance authority for payment [22] However, the chronic delay in claims payment means regular stockout of drugs or deliberate reluctance of service providers to give drugs to NHIS patients who visit their facilities [23-25] Therefore, as a coping strategy, service providers often make NHIS clients pay for drugs in order to keep their health facilities operational. The reasons for paying for consultation fees were to cover administrative expenses of the health facilities.

A range of household characteristics were found to be the key predictors of paying out-of-pocket for health care services. Households in the poor and the least poor category were strong predictors of incurring out-of-pocket health payments. This finding is consistent with previous studies conducted in Sub-Saharan Africa which reported that the burden of out-of-pocket health payments are concentrated among better-off households [26-28] This is because households who are better off are more predisposed to make such health cost payments unlike the poorest households who are likely to forego health care services when faced with a health risk $[26,29]$

Whereas payments by the insured for services or drugs covered by the NHIS is illegal, challenges with the scheme foster these kinds of behaviors. For instance, the delay in reimbursement of health facilities, the unavailability of medicines at health facilities etc. as highlighted above prompt "under the table" payments for health care [23-25] Also, it is unclear what the boundaries are for health care providers to charge for services. It is also not known whether NHIS clients are aware of services that are not covered by the NHIS. Forty-two percent of the poorest registered with the NHIS reported paying outof-pocket payments as well. This could be a deterrent to future use of health care or worse yet, contribute to low enrolment or non-renewal of NHIS memberships.

Health insurance presents an alternative to paying out-of-pocket for services and studies showed improved 
Table 6 Out-of-pocket Payments by the Insured

\begin{tabular}{|c|c|c|}
\hline Health care payment by Insurance Status & Number & Percentage (\%) \\
\hline Respondents with Valid NHIS card and seen & 1722 & 90.3 \\
\hline No Valid NHIS Card & 178 & 9.7 \\
\hline Total & 1907 & 100 \\
\hline Paid out of Pocket with valid NHIS card seen & 803 & 46.9 \\
\hline \multicolumn{3}{|l|}{ Services paid for as NHIS valid card holder } \\
\hline Consultation with Valid card seen & 1295 & 75.3 \\
\hline Card not seen & 102 & 68.5 \\
\hline \multicolumn{3}{|l|}{ NHIS valid card purchased drugs } \\
\hline Card seen & 1079 & 63.2 \\
\hline Card not seen & 111 & 75.0 \\
\hline Proportion of NHIS earning income & 998 & 56.0 \\
\hline \multicolumn{3}{|l|}{ Monthly income by NHIS clients (GHS) } \\
\hline $8-150$ & 318 & 31.9 \\
\hline $151-300$ & 238 & 23.9 \\
\hline $301-450$ & 101 & 10.1 \\
\hline $451-600$ & 120 & 12.0 \\
\hline $600+$ & 121 & 12.1 \\
\hline Total & 998 & \\
\hline Average health care costs (GHS) & 33.1 & \\
\hline Average Income of NHIS Clients (GHS) & 574.7 & \\
\hline Average Household size of NHIS clients & 4.7 & \\
\hline
\end{tabular}

access to health care for all socio-economic groups. It is however becoming clearer that patients are still paying for health services that are supposed to be in the health insurance benefit package. In South Korea, Lee and Shaw [30] reported out-of-pocket payments with health insurance ranging from 65 to $75 \%$ for the poorest quintile. This is much higher than in this study where $42 \%$ of the insured poorest quintile pay out-of- pocket for health care, nonetheless, it is substantial for the poorest. Kanmiki et al. [31] also reported that between 2010 and 2014 out of pockets payments for health services and medications for primary care declined by 63 and $62 \%$ respectively using a 5 year panel data from the Upper East Region of Ghana implying out-of-pocket payments was in the range of 37 and 38\% within the same period.

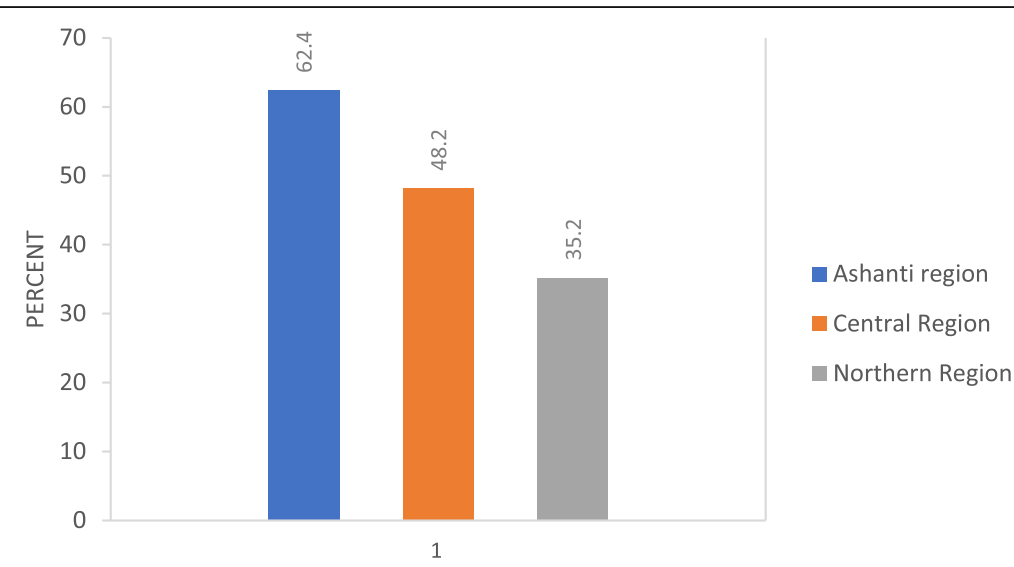

OUT-POCKET PAYMENT BY REGION

Fig. 2 Out-of-Pocket Payment by Region for Insured Clients 


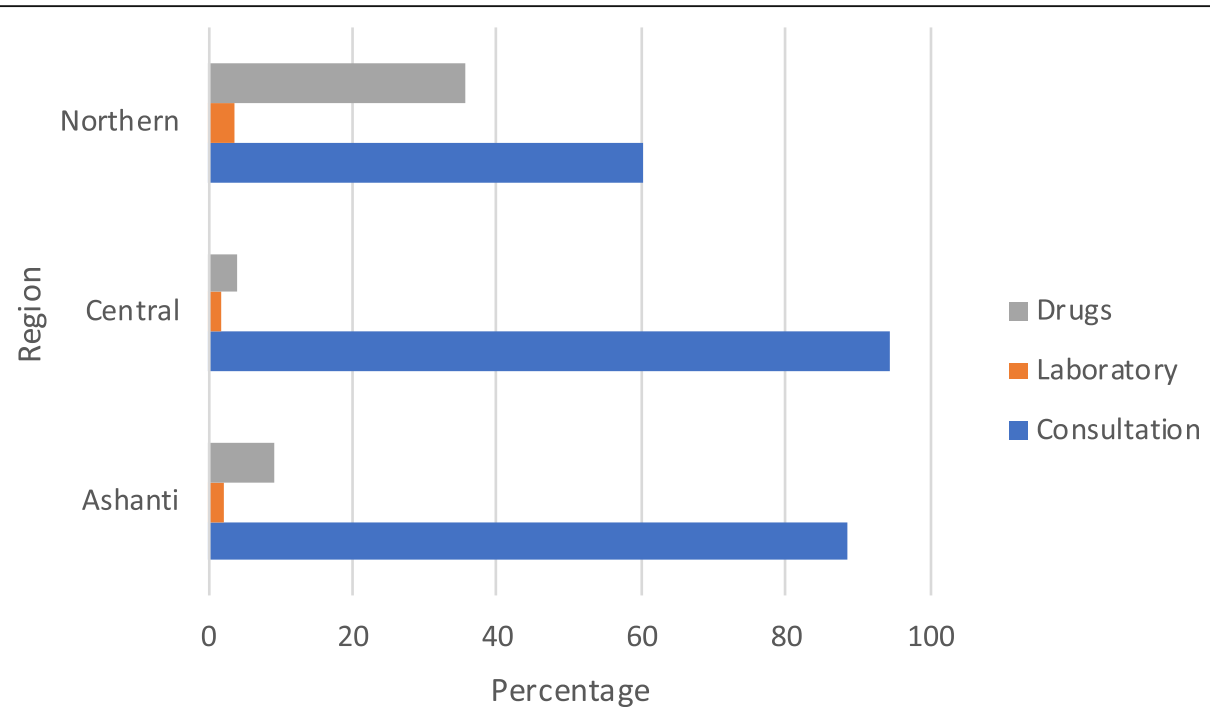

Fig. 3 Type of Services Paid Out-of-Pocket by Region (\%)

The direct out-of-pocket payments of NHIS beneficiaries continue to be substantially stable and higher relative to household incomes of clients. Direct out-ofpocket payments for the insured in Ghana is not supported by the NHIS [21] and yet it is clear that, the burden of out-of-pocket payments remains on the clients and substantially higher for those in the poorest quintile relative to their earnings.

\section{Conclusion and recommendation}

Insured clients of Ghana's NHIS seeking health care in credential health facilities make out-of-pocket payments for consultation and drugs that are covered by the scheme. The out-of-pocket payments are largely attributed to unavailability of drugs at the facilities while the consultation fees are charged to meet the administrative costs of services. These charges occur in disadvantaged regions and in all health facilities.

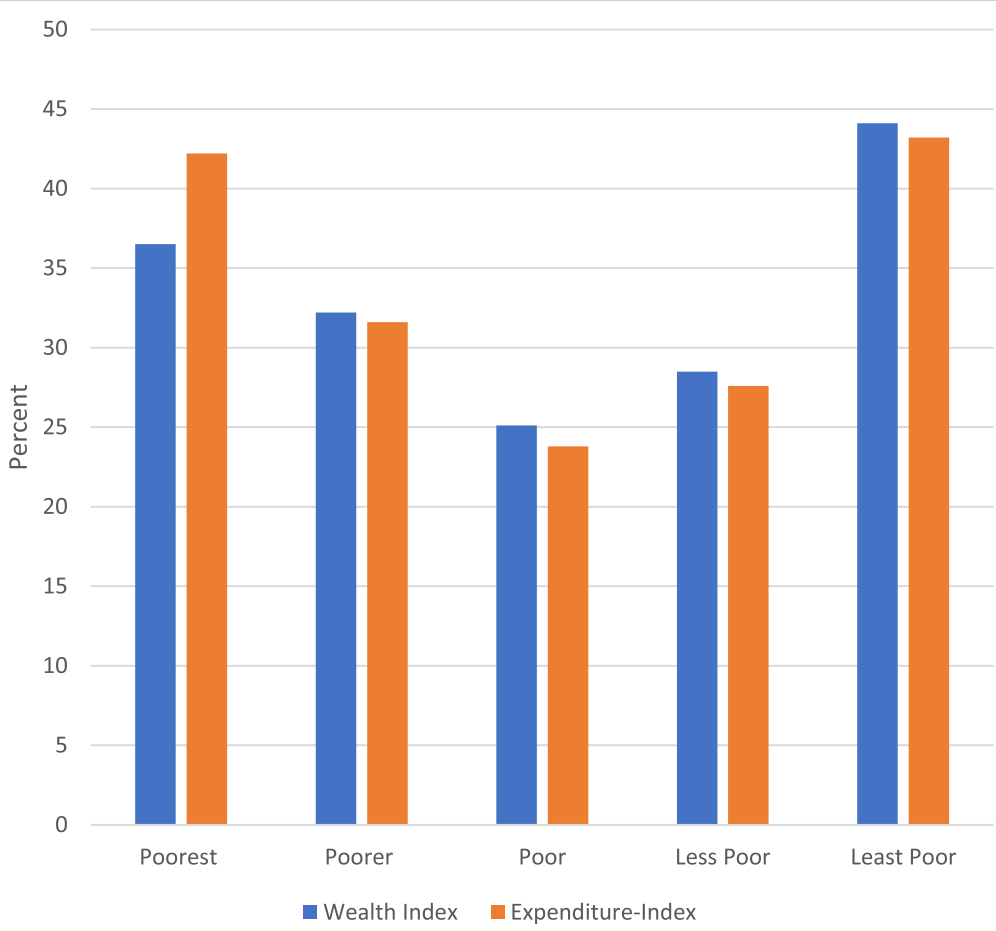

Fig. 4 Out-Of-Pocket Payment by Socio-economic Index 
Table 7 Predictors of Out-of-Pocket Payments

\begin{tabular}{|c|c|c|c|}
\hline Variable & Odds ratio & $P$-Value & $\mathrm{Cl}$ \\
\hline \multicolumn{4}{|l|}{ Sex } \\
\hline \multicolumn{4}{|l|}{ Female (B) } \\
\hline Male & 1.15 & 0.277 & $0.89-1.48$ \\
\hline \multicolumn{4}{|l|}{ Age group of Patient } \\
\hline \multicolumn{4}{|l|}{ Less than 6 years (B) } \\
\hline Age 6-17 & 1.8 & 0.406 & $0.79-1.78$ \\
\hline Age 18-49 & 1.3 & 0.131 & $0.93-1.72$ \\
\hline Age 50-59 & 1.2 & 0.272 & $0.78-1.88$ \\
\hline Age 60-69 & 0.83 & 0.454 & $0.53-1.33$ \\
\hline Age 70 and above & 1.04 & 0.853 & $0.65-1.66$ \\
\hline \multicolumn{4}{|l|}{ Level of Education } \\
\hline \multicolumn{4}{|l|}{ Never been to School (B) } \\
\hline Primary & 1.4 & $0.080^{*}$ & $0.96-2.10$ \\
\hline JHS/Middle & 1.1 & 0.429 & $0.83-1.53$ \\
\hline SHS/O/A Level & 1.23 & 0.223 & $0.88-1.72$ \\
\hline Tertiary & 1.22 & 0.343 & $0.81-1.85$ \\
\hline \multicolumn{4}{|l|}{ Region } \\
\hline \multicolumn{4}{|l|}{ Ashanti (B) } \\
\hline Central & 0.34 & $0.000^{* *}$ & $0.25-0.45$ \\
\hline Northern & 0.27 & $0.000^{* *}$ & $0.19-0.38$ \\
\hline \multicolumn{4}{|l|}{ Illness severity } \\
\hline \multicolumn{4}{|l|}{ Severe (B) } \\
\hline Moderate & 0.75 & $0.077^{*}$ & $0.53-1.03$ \\
\hline Mild & 0.66 & $0.031^{* *}$ & $0.45-0.96$ \\
\hline All forms severity & 0.83 & 0.216 & $0.62-111$ \\
\hline \multicolumn{4}{|l|}{ Type of services received } \\
\hline \multicolumn{4}{|c|}{ OPD-Folder vital statistics taken (B) } \\
\hline Laboratory investigation & 33.97 & $0.000^{* *}$ & $13.0-89.09$ \\
\hline Consultation & 12.09 & $0.0000^{* *}$ & $5.28-27.66$ \\
\hline Drugs & 8.06 & $0.000^{* *}$ & $3.33-19.51$ \\
\hline \multicolumn{4}{|l|}{ Signs-Symptoms presented } \\
\hline \multicolumn{4}{|l|}{ Malaria-like (B) } \\
\hline Difficult-Breathing & 0.99 & 0.987 & $0.69-1.43$ \\
\hline ENT-toothache & 0.59 & $0.030^{* *}$ & $0.36-0.95$ \\
\hline Injury & 1.78 & $0.027^{* *}$ & $1.06-2.96$ \\
\hline Review & 0.44 & $0.000^{* *}$ & $0.33-0.60$ \\
\hline Eye-infections & 0.42 & $0.001^{* * *}$ & $0.25-0.70$ \\
\hline Hypertension-Diabetes & 0.62 & 0.173 & $0.31-1.23$ \\
\hline Skin Infections & 0.45 & 0.183 & $0.14-1.46$ \\
\hline \multicolumn{4}{|l|}{ Poorest (B) } \\
\hline Poorer & 1.17 & 0.370 & $0.83-1.64$ \\
\hline Poor & 1.55 & $0.012^{* *}$ & $1.11-2.18$ \\
\hline Less Poor & 2.00 & $0.000^{* *}$ & $1.40-285$ \\
\hline Least Poor & 2.08 & $0.000^{* *}$ & $1.43-3.02$ \\
\hline
\end{tabular}

$\mathrm{B}$ is the base category; ${ }^{*} p<0.1$ (10\% level of Significance); ${ }^{* *} p<0.05$ (5\% level of Significance) 
The high reliance on out-of-pocket health payments can potentially impede Ghana's progress towards achieving Universal Health Coverage and Sustainable Development Goal 3 (at least 80\% essential health services coverage for the entire population of the country and $100 \%$ financial protection by the year 2030) seeking to end poverty and reduce inequalities. In order to build trust and confidence in the NHIS there is the need to eliminate out-of-pocket payments for consultation and medicines by clients.

\section{Abbreviations}

CHPS: Community-based Planning Services; GHS: Ghana Cedis; NHIS: National Health Insurance Scheme; OOP: Out-Of-Pocket; OPD: Out Patient

Department; UHC: Universal Health Coverage; USD: United States of America

\section{Acknowledgements}

We also wish to acknowledge the contributions of the following people and organization: National Health Insurance Authority, The Country Advisory Group Members and Swiss TPH and finally the study population.

\section{Authors' contributions}

The Out-of-pocket study was designed by PA in collaboration with MA and FT. The manuscript was initiated by PA, MA. FT, KW and PS read and reviewed the manuscript. Field work for this initiative was organized by PA and MA. Initial data analysis was conducted by PA, PS, MA, and FT. The authors read and approved the final manuscript.

\section{Funding}

The manuscript is part of the research project 'Health systems governance for an inclusive and sustainable social health protection in Ghana and Tanzania' funded by the Swiss Program for Research on Global Issues for Development. This is a joint programme by the Swiss National Science Foundation (SNSF) and the Swiss Agency for Development and Cooperation (SDC). The funder of the study had no role in the study design, data gathering, analysis and interpretation, or in writing of the report. The corresponding author had full access to all the data in the study and had final responsibility for the decision to submit for publication.

\section{Availability of data and materials}

The data is part of an ongoing study. In view of that we are restricted from sharing the large data set. However, the data contained in this manuscript are available upon a reasonable request to the corresponding author.

\section{Declaration}

\section{Ethics approval, guidelines and consent to participate}

All the methods undertaken to collect data from participants were carried out in accordance with the relevance guidelines and regulations of the Ethics Committee. Care was taken to report data in the aggregate to ensure confidentiality of the participant details. Participation in the study was voluntary and participants were able to withdraw from the study at any point in time. For minors, informed consent was obtained from parents or caregivers who accompanied them to the health facility. Informed consent was sought from all adult participants 18 years and above.

The protocol for the study was reviewed and approved (GHS-ERC: 012/03/

18) by the Ghana Health Service Ethics Review Committee.

\section{Consent for publication}

Not Applicable

\section{Competing interests}

The authors declare that they have no competing interests.

\section{Author details}

'School of Public Health, University of Ghana, P. O. Box LG13, Accra, Ghana. ${ }^{2}$ Swiss Tropical and Public Health Institute (Swiss TPH), Basel, Switzerland, University of Basel, Basel, Switzerland, Socintrasse 57, 4051 Basel, Switzerland.
${ }^{3}$ Institute of Pharmaceutical Medicine (ECPM), University of Basel, Petersplatz 1, 4001 Basel, Switzerland.

Received: 21 December 2020 Accepted: 12 April 2021

Published online: 08 May 2021

\section{References}

1. Wagstaff A, Flores G, Hsu J, Smitz MF, Chepynoga K, Buisman LR, et al. Progress on catastrophic health spending in 133 countries: a retrospective observational study. Lancet Glob Heal. 2018;6(2):e169.

2. World Health Organization. World Health Report: Health Systems Financing: The Path to Universal Coverage. Geneva: World Heal Organization; 2010. p. 1-17.

3. Garcia-Subirats I, Vargas I, Mogollón-Pérez AS, De Paepe P, Da Silva MRF, Unger JP, et al. Inequities in access to health care in different health systems: a study in municipalities of central Colombia and north-eastern Brazil. Int J Equity Health. 2014;13(1):10.

4. Sood N, Bendavid E, Mukherji A, Wagner Z, Nagpal S, Mullen P. Government health insurance for people below poverty line in India: quasi-experimental evaluation of insurance and health outcomes. BMJ. 2014;349(sep25 4): g5114. https://doi.org/10.1136/bmj.g5114.

5. Kusi A, Enemark U, Hansen KS, Asante FA. Refusal to enrol in Ghana's National Health Insurance Scheme: is affordability the problem? Int J Equity Health. 2015;14(1):2. https://doi.org/10.1186/s12939-014-0130-2.

6. Chang FR, Trivedi K, Economics P. Of self-medication: theory and evidence. Health Econ. 2003;12(9):721-39. https://doi.org/10.1002/hec.841.

7. Nguyen $\mathrm{CV}$. The impact of voluntary health insurance on health care utilization and out-of-pocket payments: new evidence for Vietnam. Health Econ. 2012;21(8):946-66. https://doi.org/10.1002/hec.1768.

8. Sepehri A, Sarma S, Simpson W. Does non-profit health insurance reduce financial burden? Evidence from the Vietnam living standards survey panel. Health Econ. 2006;15(6):603-16. https://doi.org/10.1002/hec.1080.

9. Nsiah-Boateng E, Aikins M. Trends and characteristics of enrolment in the National Health Insurance Scheme in Ghana: a quantitative analysis of longitudinal data. Glob Heal Res Policy. 2018;3(1):32. https://doi.org/10.1186/ s41256-018-0087-6.

10. Navarrete F, Ghislandi S, Stuckler D, Tediosi F. Inequalities in the benefits of national health insurance on financial protection from out-of-pocket payments and access to health services: cross-sectional evidence from Ghana. Heal Policy Plan. 2019;34(9):594-705.

11. Alhassan RK, Duku SO, Janssens W, Nketiah-Amponsah E, Spieker N, van Ostenberg $\mathrm{P}$, et al. Comparison of perceived and technical healthcare quality in primary health facilities: implications for a sustainable National Health Insurance Scheme in Ghana. PLoS One. 2015;10:e0140109.

12. Atinga AR. Healthcare quality under the National Health Insurance Scheme in Ghana: perspectives from premium holders. Int J Qual Reliab Manag. 2012;29(2):144-61. https://doi.org/10.1108/02656711211199883.

13. Dalinjong PA, Wang AY, Homer CS. Has the free maternal health policy eliminated out of pocket payments for maternal health services? Views of women, health providers and insurance managers in northern Ghana. PLoS One. 2018;13(2):e0184830. https://doi.org/10.1371/journal.pone.0184830.

14. Duku SKO, Nketiah-Amponsah E, Janssens W, Pradhan M Perceptions of healthcare quality in Ghana: Does health insurance status matter? PLoS ONE. 2018;13(1)

15. Macha J, Harris B, Garshong B, Ataguba JE, Akazili J, Kuwawenaruwa A, Borghi J. Factors influencing the burden of health care financing and the distribution of health care benefits in Ghana, Tanzania and South Africa. Health Policy Plan. 2012;27(Suppl 1):i46-54.

16. National Health Insurance Authority. National Health Insurance AuthorityAnnual Report. Accra: National Health Insurance Authority; 2017.

17. Ghana Minimum Wage. Wagelndicator 2018 - Wagelndicator.org - Ghana.

18. Ghana Health Service. Ghana Health Service-Annual Report. Accra: Ghana Heal Service; 2019

19. Abuosi AA, Adzei FA, Anarfi J, Badasu DM, Atobrah D, Yawson A. Investigating parents/caregivers financial burden of care for children with non-communicable diseases in Ghana. BMC Pediatr. 2015;

20. Aryeetey GC, Westeneng J, Spaan E, Jehu-Appiah C, Agyepong IA, Baltussen R. Can health insurance protect against out-of-pocket and catastrophic expenditures and also support poverty reduction? Evidence from Ghana's National Health Insurance Scheme. Int J Equity Health. 2016;15(1):116.

21. Ghana Statistical Service. Poverty Trends In Ghana (2005-2017). Ghana: Ghana Statistical Service; 2018. 
22. Ministry of Health. National Health Insurance Policy Framework for Ghana. Accra: Minist Heal Ghana; 2004

23. Agyepong IA, Nagai R. We charge them; otherwise we cannot run the hospital front line workers, clients and health financing policy

implementation gaps in Ghana. Health Policy (New York). 2011;99:226-333 Available from: https://doi.org/10.1016/j.healthpol.2010.09.018.

24. Dalinjong PA, Laar AS. The national health insurance scheme: perceptions and experiences of health care providers and clients in two districts of Ghana. Heal Econ Rev [Internet]. 2012; Available from: https://doi.org/10.11 86/2191-1991-2-13.

25. Sodzi-Tettey S, Aikins M, Awoonor-Williams JK, Agyepong IA. Challenges in provider payment under the Ghana National Health Insurance Scheme: a case study of claims management in two districts. Ghana Med J. 2012;46(4): 189-99.

26. Masiye F, Kaonga O. Determinants of healthcare utilisation and out-ofpocket payments in the context of free public primary healthcare in Zambia. Int J Heal Policy Manag. 2016;5(12):693-703. https://doi.org/10.151 71/ijhpm.2016.65.

27. Onwujekwe OE, Uzochukwu BS, Obikeze EN, Okoronkwo I, Ochonma OG, Onoka CA, et al. Investigating determinants of out-of-pocket spending and strategies for coping with payments for healthcare in Southeast Nigeria. BMC Heal Serv Res. 2010; 10: (67). Available from: https://doi.org/10.1186/14 72-6963-10-67, 2010

28. Wang W, Temsah G, Carter E. Levels and determinants of out-of-pocket health expenditures in the Democratic Republic of the Congo, Liberia, Namibia, and Rwanda. 2016;

29. McIntyre D, Thiede M, Dahlgren G, Whitehead M. What are the economic consequences for households of illness and of paying for health care in low- and middle-income country contexts? Soc Sci Med. 2006;62:858-65 Available from: https://doi.org/10.1016/j.socscimed.2005.07.001.

30. Lee WY, Shaw I. The impact of out-of-pocket payments on health care inequity: the case of national health insurance in South Korea. Int J Environ Res Public Health. 2014;11(7):7304-18. https://doi.org/10.3390/ijerph110707304.

31. Kanmiki E, Bawah A, Phillips J, Awoonor-Williams J, Kachur S, Asuming P, et al. Out-of-pocket payment for primary healthcare in the era of national health insurance: evidence from northern Ghana. PLoS One. 2019;14(8): e0221146 https://doi.org/10.1371/journal.pone.022.

\section{Publisher's Note}

Springer Nature remains neutral with regard to jurisdictional claims in published maps and institutional affiliations.

Ready to submit your research? Choose BMC and benefit from:

- fast, convenient online submission

- thorough peer review by experienced researchers in your field

- rapid publication on acceptance

- support for research data, including large and complex data types

- gold Open Access which fosters wider collaboration and increased citations

- maximum visibility for your research: over $100 \mathrm{M}$ website views per year

At $\mathrm{BMC}$, research is always in progress.

Learn more biomedcentral.com/submissions 